\title{
HIPERTENSÃO INTRACRANIANA
}

\author{
INTRACRANIAL HYPERTENSION
}

Carlos G. Carlotti Jr¹; Benedicto O. Colli \& Luiz A. A. Dias²

\begin{abstract}
${ }^{1}$ Docente do Departamento de Cirurgia, Ortopedia e Traumatologia da Faculdade de Medicina de Ribeirão Preto - USP, Disciplina de Neurocirurgia. ${ }^{2}$ Médico Assistente do Departamento de Cirurgia, Ortopedia e Traumatologia da Faculdade de Medicina de Ribeirão Preto - USP e Chefe do Serviço de Neurocirurgia da Santa de Misericórdia de Ribeirão Preto.

CorResPondÊnCIA: Carlos Gilberto Carlotti Junior - Departamento de Cirurgia, Ortopedia e Traumatologia do Hospital das Clínicas FMRP - Campus Universitário USP - CEP: 14048-900 - Ribeirão Preto - SP - FAX - (016) 602-2498 - e-mail - cgcjunio@fmrp.usp.br
\end{abstract}

CARLOTTI JR CG; COLLI BO \& DIAS LAA. Hipertensão intracraniana. Medicina, Ribeirão Preto, 31: 552-562, out./dez. 1998.

RESUMO: A relação entre o conteúdo da caixa intracraniana e o seu volume determina a pressão intracraniana (PIC), que tem como referência a pressão atmosférica. Em condições normais, a pressão intracraniana tem flutuações determinadas pelos ciclos respiratório e cardíaco. Várias doenças determinam o aumento da pressão intracraniana, sendo a mais freqüente o traumatismo craniencefálico.

Para o diagnóstico da hipertensão intracraniana (HIC) deve-se valorizar o quadro clínico, constituído de cefaléia, vômitos e papiledema. Dos exames subsidiários, os mais importantes são os métodos de imagem principalmente Tomografia Computadorizada (TC) e Ressonância Nuclear Magnética (RNM).

Para os casos graves de HIC, o ideal durante o tratamento é que a PIC esteja monitorizada. Diversas modalidades podem ser utilizadas, como a hipocapnia induzida pela hiperventilação, os diuréticos osmóticos, a hipotermia e cuidados especiais no tratamento geral dos pacientes.

UNITERMOS: Pressão Intracraniana. Diagnóstico.

\section{INTRODUÇÃO}

Pressão intracraniana (PIC) é aquela encontrada no interior da caixa craniana, tendo como referência a pressão atmosférica ${ }^{(1)}$. A PIC tem uma variação fisiológica de 5 a $15 \mathrm{mmHg}^{(2)}$ e reflete a relação entre o conteúdo da caixa craniana (cérebro, líquido cefalorraquidiano e sangue) e o volume do crânio, que pode ser considerado constante (Doutrina de Monroe-Kellie). A alteração do volume de um desses conteúdos pode causar a hipertesão intracraniana (HIC).

\section{FISIOPATOLOGIA}

O líquido cefalorraquidiano (LCR) constitui $10 \%$ do volume intracraniano e seu volume, em todo o sistema nervoso, é de aproximadamente $150 \mathrm{ml}$, dos quais 20-30 ml estão no interior dos ventrículos e o restante nos espaços subaracnóides intracraniano e raquidiano.
O LCR é produzido em torno de $0,3 \mathrm{a} 4,0 \mathrm{ml} / \mathrm{min}$, principalmente nos plexos coróideos dos ventrículos laterais (70\% da produção), e, em menor quantidade, por transudação de líquido através do epêndima ${ }^{(3,4)}$.

Uma vez vez produzido, o LCR dos ventrículos laterais circula através dos forames de Monro para o terceiro ventrículo e daí para o quarto ventrículo, através do aqueduto cerebral. Do quarto ventrículo, o LCR sai pelos forames de Luschka e Magendie e alcança as cisternas basais. Por via anterior, através das cisternas anteriores do tronco cerebral, alcança a convexidade do cérebro, após passar pela base dos lobos frontais e temporais. Por via posterior, o LCR que sai do quarto ventrículo circula pela cisterna magna, cisternas supracerebelares, cisternas ambientes e cisternas do corpo caloso, atingindo também a convexidade cerebral. Além disso, o LCR circula ao redor da medula no canal raquidiano, em um movimento de entrada e saída na caixa craniana. A propagação da 
corrente liquiórica é atribuída ao efeito exercido pelas pulsações cardíacas nas artérias do plexo coróideo, o qual provoca uma onda de pressão $0^{(5,6)}$.

A reabsorção liquórica ocorre, em grande parte, nas vilosidades aracnóideas, ao longo do seio sagital, através de um mecanismo passivo do tipo valvular unidirecional. Quando a pressão liquórica atinge $5 \mathrm{~mm} / \mathrm{H}_{2} \mathrm{O}$, mecanismos valvulares nos canalículos que unem o espaço subaracnóideo às veias que drenam para o seio sagital superior abrem-se e permitem o escoamento do LCR, para dentro do sistema venoso.

As alterações liquóricas que levam à HIC, geralmente, são aquelas que causam obstrução da circulação liquórica em qualquer ponto de sua via e as que causam dificuldade na reabsorção do LCR.

O volume total de sangue intracraniano é, aproximadamente, 4-4,5 ml/100 g de tecido cerebral, que, normalmente, está distribuído em $60 \%$ no lado venoso e $40 \%$ no lado arterial. Clinicamente, o sistema venoso pode ser considerado incompressível e os vasos do sistema venoso não sofrem alterações dos seus diâmetros, portanto toda a resposta vascular cerebral está do lado arterial, que representa menos de $2 \%$ do volume intracraniano ou aproximadamente $25 \mathrm{ml} \mathrm{no}$ cérebro adulto.

O fluxo sangüíneo cerebral (FSC) é diretamente proporcional à pressão de perfusão cerebral (PPC) e inversamente proporcional à resistência vascular cerebral (RVC). A PPC é igual a pressão arterial média (PAM) menos a pressão venosa. Como, no homem, a pressão nos seios venosos é difícil de ser medida e ela corre paralela à PIC, considera-se a PPC igual à diferença entre a PAM e a PIC. Portanto, o FSC pode ser expresso na seguinte equação:

$$
\mathrm{FSC}=\frac{\mathrm{PPC}}{\mathrm{RVC}}=\frac{\mathrm{PAM}-\mathrm{PV}(\mathrm{PIC})}{\mathrm{RVC}}
$$

A auto-regulação do FSC pode ser definida como a capacidade de aumento do FSC devido ao aumento da necessidade metabólica do cérebro ${ }^{(7)} \mathrm{e}$ diminuição do fluxo com redução da demanda (auto-regulação metabólica) ou como a capacidade de manutenção do fluxo apesar do aumento ou da redução da pressão arterial sistêmica (auto-regulação pressórica) ${ }^{(8)}$.

A auto-regulação funciona adequadamente na faixa de variação da PAM, de 50 a $160 \mathrm{mmHg}$, o que significa que, conforme a PAM diminui, os vasos de resistência dilatam até que atinjam um ponto máximo em resposta à redução da pressão. A partir de 50 mmHg, o FSC reduz abruptamente com quedas adicionais da PAM. O resultado dessa intensa vasodilatação é um quadro de vasoplegia capilar, que provoca ingurgitamento da microcirculação. Essa vasoplegia pode ser irreversível e, com o aumento progressivo, a PIC pode igualar-se à PAM, interrompendo o $\mathrm{FSC}^{(1)}$.

Com o aumento da PAM, os vasos contraem-se até que a PAM atinja $160 \mathrm{mmHg}$, nível em que a pressão quebra a resistência da vasoconstrição, causando dilatação passiva e um aumento no FSC.

A teoria mais aceita para a regulação do FSC é a metabólica, que se baseia na premissa de que os músculos das paredes vasculares são influenciados por metabólitos vasodilatadores, produzidos nos tecidos vizinhos. $\mathrm{O} \mathrm{CO}_{2}$ tem um acentuado efeito relaxante na musculatura dos vasos cerebrais e, conseqüentemente, suas alterações têm um grande efeito sobre a resistência vascular, sobre o FSC e sobre o volume sangüíneo cerebral. Entretanto seu efeito não é direto sobre o diâmetro das arteríolas e, sim, age mediado pela alteração que provoca no $\mathrm{pH}$ do líquido extracelular ${ }^{(8)}$.

Em resumo, o acúmulo de $\mathrm{CO}_{2}$ no espaço intersticial leva à acidose tecidual, que ocasiona o relaxamento da musculatura lisa da microcirculação e reduz a RVC. O contrário ocorre quando o $\mathrm{CO}_{2}$ é eliminado e o pH tecidual aumenta. Embora com a auto-regulação o FSC permaneça constante, o fluxo sangüíneo regional varia de acordo com as necessidades metabólicas regionais, havendo uma relação direta entre o fluxo e a atividade metabólica das áreas do córtex cerebral.

Uma extensão do conceito de auto-regulação cerebral é o de acoplamento, que é a relação ideal do FSC com o metabolismo tissular. A condição patológica é chamada de desacoplamento, quando pode ocorrer excesso de FSC para um tecido que não está consumindo toda a oferta oferecida (hiperemia) ou um FSC menor que as necessidades do tecido (oliguemia); tais situações têm sido descritas no traumatismo craniencefálico (TCE). Uma forma de avaliar esse acoplamento é o de medir a diferença da saturação arteriovenosa (jugular) de $\mathrm{O}_{2}$ e inferir se o tecido cerebral está extraindo muito oxigênio (déficit de fluxo) ou se existe oxigênio em excesso no sangue venoso (excesso de fluxo) $)^{(8,9,10)}$.

A pressão venosa intracraniana também deve ser considerada no estudo da etiologia da HIC, doenças intracranianas (fístulas arteriovenosas) e extracranianas (obesidade mórbida, trombose traumática de veias jugulares) podem causar seu aumento e, secundariamente, HIC ${ }^{(11 / 15)}$. 
O parênquima cerebral contribui com $85 \%$ do volume intracraniano (1000 a $1250 \mathrm{ml})$, sendo constituído por substância branca, onde há predomínio de axônios e mielina, e por substância cinzenta, onde há predomínio de corpos celulares. A parte sólida representa $25 \%$ do parênquima e os $75 \%$ restantes são constituídos por água, distribuída nos espaços extra e intracelulares ${ }^{(516)}$.

O aumento do volume cerebral pode se dar pelo crescimento anormal de um tecido (tumores), pelo aparecimento de uma resposta inflamatória em resposta a um agente infeccioso (abscesso ou granulomas) ou pelo acúmulo de líquido nos espaços intersticial e/ou intracelular. $\mathrm{O}$ acúmulo é chamado de edema cerebral, e resulta do funcionamento indequado dos mecanismos de transporte da água e de eletrólitos entre os capilares e espaços extra e intracelular. De acordo com o mecanismo de formação, pode ser dividido em vasogênico e citotóxico.

O edema vasogênico resulta da quebra da barreira hematoencefálica, o que provoca aumento da permeabilidade do endotélio capilar e permite o extravasamento de componentes do plasma (água e proteínas), para o espaço intersticial. A partir do local da lesão, o líquido do edema espalha-se pela substância branca adjacente, por gradiente de pressão. A composição do líquido do edema é intermediária entre o plasma e o líquido extracelular e varia desde a sua formação até a sua resolução ${ }^{(5,17,18)}$.

Os principais efeitos prejudiciais do edema vasogênico são: alteração na perfusão tissular por dificuldade de fluxo nos capilares, aumento do volume tissular, dificultando a difusão adequada de substratos metabólicos e catabólitos entre os compartimentos tissular e vascular, desmielinização ou interrupção do fluxo axoplasmático secundários à pressão sobre os axônios, e alterações na excitabilidade da membrana nervosa pelos componentes do líquido do edema ${ }^{(1)}$.

O edema citotóxico, ou celular, é o acúmulo de líquido no espaço intracelular, caracterizado por um aumento do volume intracelular e redução do volume extracelular. O mecanismo ultra-estrutural do edema celular é a alteração da membrana celular. A situação clínica em que ele ocorre com maior freqüência é a isquemia, por alteração no suporte energético das trocas iônicas ao nível da membrana, tanto no neurônio como nas células da glia. Segundos após a falência da bomba de $\mathrm{Na}^{+}$e $\mathrm{K}^{+}$, verifica-se o acúmulo de água e $\mathrm{Na}^{+}$dentro da célula e de $\mathrm{K}^{+}$no líquido extracelular, cujo grau depende da duração e intensidade da isque- mia. Nessa fase, não há quebra da barreira hematoencefálica. Entretanto, durante a evolução do edema celular por isquemia, ocorre a sua tranformação em vasogênico. A reversão do edema intracelular pode ocorrer quando os mecanismos de troca iônica ativa, na membrana celular são restabelecidos ${ }^{(19,20)}$.

Além desses dois tipos, são ainda descritos o edema hidrostático, que ocorre no espaço intersticial por aumento da pressão venosa (edema passivo), e o denominado edema intersticial, que se verifica nas regiões periventriculares, pela transudação transependimária de LCR, em pacientes com hidrocefalia e HIC.

O edema pode levar a um aumento da PIC com conseqüente redução do FSC, o que, por sua vez, leva à hipóxia, que contribui para o aumento do edema, fechando um círculo vicioso. O círculo vicioso, se não for impedido pelos mecanismos normais de reabsorção ou por medidas terapêuticas, leva à interrupção do FSC, que é o principal parâmetro clínico para a determinação da morte cerebral.

A relação do volume intracraniano com a PIC não se faz de forma linear, mas, sim, exponencial. A injeção de pequenos volumes de líquido no interior do crânio, de início, praticamente não altera a PIC, nas injeções subseqüientes, o aumento é lento, mas, a partir de um volume injetado, o acréscimo de pequenos volumes de líquidos determina grandes aumentos na PIC e vice-versa. Esse fato acontece devido aos mecanismos tampões, existentes no interior do crânio, ou seja, a saída de líquido cefalorraquidiano para dentro do saco dural ou sua reabsorção ( $70 \%$ da capacidade de compensação intracraniana) e a redução do volume sangüíneo por compressão do leito vascular e ejeção do sangue para fora da caixa craniana ( $30 \%$ da capacidade de compensação espacial). Esta relação está representada na Figura $1^{(21)}$.

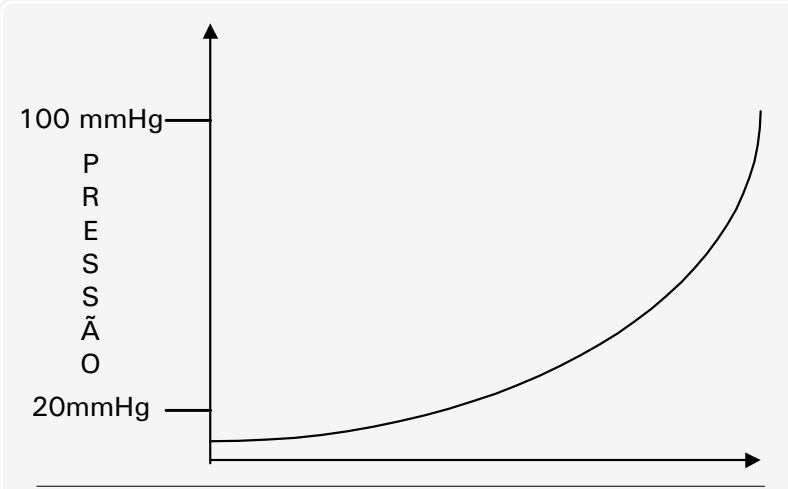

Figura 1 - Curva da relação Pressão/Volume da Pressão Intracraniana 


\section{MANIFESTAÇÕES CLÍNICAS}

As manifestações clássicas da HIC, nos adultos e nas crianças maiores, são a cefaléia, as alterações visuais e as náuseas e vômitos. Outros sinais que podem ser observados são os ditúrbios psíquicos, paresia do VI nervo craniano (desvio medial do olho), que não tem valor como sinal localizatório, e tonturas ${ }^{(1,2,18,22,23)}$.

A cefaléia ocorre pelo aumento da pressão e por distensão da dura-máter, dos vasos e dos nervos cranianos, que são estruturas que têm terminações nervosas sensitivas. Geralmente, é mais freqüente no período da manhã e acorda o paciente durante a noite, e pode melhorar durante o dia, quando o paciente permanece na posição supina o que facilita o retorno venoso.

Os vômitos são devidos ao aumento da pressão e à irritação do assoalho do quarto ventrículo. Além disso, podem ocorrer tonturas e alterações discretas da marcha.

No exame clínico, é importante o exame do fundo de olho para detectar o papiledema. O edema de papila ocorre por propagação retrógrada da hipertensão pelo espaço subaracnóideo ao redor do nervo óptico, que funciona como um manguito e dificulta o retorno venoso pela veia oftálmica que tem um trajeto parcial dentro do nervo. A cefaléia e os vômitos são comuns a muitas outras doenças mas o edema de papila é um sinal que, na maioria das vezes, indica HIC.

Nos recém-nascidos e lactentes, devido a não soldadura das suturas, esses sinais não são observados e as manifestações clínicas apresentadas, são abaulamento da fontanela, irritabilidade, macrocrania e outras alterações, como choro fácil e recusa da alimentação.

A HIC pode determinar outros sinais e sintomas que resultam das herniações do tecido cerebral e do deslocamento cefalocaudal do tronco cerebral, geralmente causados por lesões expansivas supratentoriais. Essa sintomatologia caracteriza o quadro de descompensação da HIC.

A herniação subfálcica ocorre quando há crescimento de lesão expansiva em um dos hemisférios cerebrais, deslocando o giro do cíngulo por sob a borda livre da foice do cérebro. Isso pode causar a compressão das artérias pericalosas, com infarto de seus territórios, resultando em paresia de um ou de ambos os membros inferiores.

As lesões supratentoriais hemisféricas, como edema difuso ou localizado, tendem a deslocar o tron- co cerebral no sentido cefalocaudal e provocar distorções e isquemias no diencéfalo, cujas manifestações clínicas caracterizam a denominada herniação do tipo central. O sofrimento do diencéfalo inicia pela alteração na capacidade de concentração e da memória recente e, posteriormente, pelo comprometimento do nível de consciência, por aparecimento de pupilas pequenas, além de manifestações de lesão do trato piramidal, geralmente do tipo postura de decorticação. A respiração é caracterizada por inspirações profundas, com pausas ocasionais; muitos pacientes apresentam respiração do tipo Cheyne-Stokes ${ }^{(24)}$.

Quando a compressão é ocasionada por herniação do uncus do lobo temporal, lateralmente, através da incisura, o sofrimento do nervo oculomotor manifesta-se, inicialmente, pela dilatação da pupila homolateral, que também perde reatividade à luz, e, em seguida, pela paralisia na musculatura extrínsica do globo ocular. A compressão do pedúnculo cerebral (mesencéfalo), caracteriza-se pelo aparecimento de manifestações de lesão do trato piramidal do tipo reação de descerebração, evidenciada do lado oposto à midríase. Observa-se também alteração do nível de consciência por mecanismo semelhante ao referido na herniação central. A artéria cerebral posterior pode ser comprimida pela hérnia lateral, o que pode causar infarto na região occipital e ocasionar alterações do campo visual, que, geralmente, são difíceis de serem detectadas na fase aguda. A progressão de lesão leva ao sofrimento mesencefálico bilateral, com a conseqüente extensão do quadro clínico ${ }^{(24)}$.

As lesões expansivas frontais e occipitais provocam a hernição da porção posterior do uncus do lobo temporal na região posterior da incisura, comprimindo diretamente o teto do mesencéfalo. Os sinais clínicos que caracterizam a herniação posterior são a síndrome de Parinaud, ptose palpebral bilateral, e flexão da cabeça, além de alterações do nível de consciência.

O deslocamento cefalocaudal do tronco cerebral pode provocar a tração dos nervos oculomotores e, algumas vezes, torna-se difícil a separação dos quadros clínicos devido à herniação central e à herniação lateral.

As lesões pontinas apresentam-se com o aparecimento de pupilas mióticas, às vezes puntiformes, e quando o bulbo é atingido, aparecem as alterações dos sinais vitais. Porém, nas herniações transtentoriais, não se observam as pupilas puntiformes como nas lesões pontinas e, sim, pupilas médio-fixas ou midriáticas, 
porque ocorre lesão concomitante das fibras parassimpáticas pupiloconstritoras. O comprometimento do bulbo pode ocorrer por lesões isquêmicas, causadas por distorção do tronco cerebral ou por compressão direta, causada por herniação das amígdalas cerebelares ${ }^{(24)}$.

A Tabela I apresenta um resumo dos tipos de herniação do parênquima cerebral.

A resposta de Cushing, caracterizada por um aumento reflexo da pressão arterial, bradicardia e alterações do ritmo respiratório na vigência de HIC, é fenômeno inconstante que parece estar relacionado com a gravidade da hipertensão, ou seja, aparece em fases avançadas da descompensação da hipertensão e constitui uma situação muito grave, com evolução para a morte, a não ser que a PIC seja rapidamente reduzida com medidas terapêuticas apropriadas. Esse fenômeno é atribuído a um aumento da resistência vascular sistêmica a um aumento do débito cardíaco, causados por influxos autonômicos, provenientes do tronco cerebral isquemiado ou comprimido, ou causado pela liberação de substâncias simpaticomiméticas, a partir de centros vasopressores do tronco cerebral.

A ocorrência de edema pulmonar em pacientes com TCE grave é conhecida desde há muito tempo e procura-se relacionar o fenômeno ao aumento da PIC.
Uma explicação para o fato é a ocorrência de influxos autonômicos, desde o bulbo, para os vasos pulmonares, resultando em edema, que é de origem primariamente neurogênica. Outra explicação é que a HIC produz uma sobrecarga no ventrículo esquerdo, que começa a falhar, e a elevação da pressão no átrio esquerdo causa aumento da pressão hidrostática na microcirculação pulmonar, com transudação de líquido, desde o leito capilar, para os alvéolos.

\section{MEDIDA DA PRESSÃO INTRACRANIANA}

A medida real da PIC é sempre invasiva e sua indicação depende de uma avaliação de risco/benefício para o paciente. Em algumas situações, ela é necessária, como no traumatismo craniano grave e a HIC é suspeitada, seja pela Tomografia Computadorizada ou por um quadro clínico grave, com um valor da Escala de Coma de Glasgow inferior a 8. Em outras situações, ela pode ser usada ou não, como nos casos graves de isquemia cerebral, pós-operatório de neurocirurgia, meningite grave, encefalite e monitorização de pacientes com problemas em sistemas de válvulas empregadas no tratamento de hidrocefa$\operatorname{lia}^{(1,2,23,25,26)}$.

\begin{tabular}{|c|c|c|}
\hline Local da Compressão & Sintomatologia & Estrutura Herniada \\
\hline Artéria Cerebral Anterior & $\begin{array}{l}\text { Pouco Freqüentes (Monoparesia ou Diplegia Crural, } \\
\text { Hemianestesia) }\end{array}$ & $\begin{array}{l}\text { Giro do Cíngulo sob a Foi- } \\
\text { ce Cerebral }\end{array}$ \\
\hline Artéria Cerebral Posterior & $\begin{array}{l}\text { Hemianópsia } \\
\text { Amaurose }\end{array}$ & $\begin{array}{l}\text { Região Póstero-Medial do } \\
\text { Lobo Temporal }\end{array}$ \\
\hline Diencéfalo & $\begin{array}{l}\text { Alteração do Nível de Consciência; Pupilas Pequenas } \\
\text { Reagentes; Hemiparesia Ipsolateral ou Tetraparesia; } \\
\text { Atitude de Decorticação; Sinal de Babinski Contra ou } \\
\text { Bilateral; Respiração de Cheyne-Stokes }\end{array}$ & $\begin{array}{l}\text { Regiões Ântero-Mediais do } \\
\text { Lobo Occipital }\end{array}$ \\
\hline Mesencéfalo & $\begin{array}{l}\text { Dilatação Pupilar Ipsolateral ou Bilateral (tardia); (Pu- } \\
\text { pilas do Médio Fixas - Teto do Mesencéfalo); Alteração } \\
\text { do Nível de Consciência; Paralisia Oculomotora Exter- } \\
\text { na, Ipsolateral (Tardia); Hemiparesia Contralateral ou } \\
\text { Tetraparesia (Tardia); Atitude de Descerebração Contra } \\
\text { ou Bilateral (Tardia); Sinal de Babinski Contralateral; } \\
\text { Hiperventilação }\end{array}$ & Uncus do Lobo Temporal \\
\hline Bulbo & $\begin{array}{l}\text { Alteração do Nível de Consciência; Respiração Lenta / } \\
\text { Atáxica Lenta / Apnéia; Redução do Ritmo Cardíaco e } \\
\text { da Pressão Arterial; Parada Cárdiorrespiratória }\end{array}$ & Tonsilas Cerebelares \\
\hline
\end{tabular}


Uma medida isolada da PIC não é adequada para a sua avaliação. A pressão medida através da agulha no canal raquidiano ou na cisterna magna pode ser incorreta na vigência de um bloqueio ao nível da incisura, o qual pode impedir a livre transmissão da PIC. Por outro lado, na vigência de um bloqueio, a punção pode desencadear um cone de pressão e o deslocamento cefalocaudal do tronco cerebral. A medida isolada da PIC, no interior do ventrículo, também pode não refletir a sua realidade, devido às variações que esta pode sofrer ao longo do dia.

A maneira ideal de avaliação da PIC é através do seu registro contínuo, pois dessa forma podem ser observadas não somente as suas variações quantitativas, mas, também, o aspecto morfológico do traçado, que, por si só, pode indicar anormalidades. A PIC pode ser monitorizada através do estabelecimento de uma coluna líquida entre o LCR e um transdutor de pressão, através da implantação de um transdutor no espaço epidural, subaracnóideo ou intraventricular ou através da implantação de um transdutor sobre a fontanela bregmática, em recém-nascidos.

Esses métodos apresentam, como vantagem, a transmissão natural da pressão através da coluna líquida, a possibilidade do uso dos transdutores comuns e a possibilidade de remoção de LCR para alívio da hipertensão ou para análise. Como inconvenientes, a coluna líquida favorece a infecção pelo contato do meio interno com o externo e, raramente, observamse hematomas no trajeto da cânula ventricular; mas, os problemas mais freqüentes, são as obstruções do cateter pelo tecido cerebral. Para a monitorização intraventricular, o ventrículo não pode estar colabado ou deformado pelo processo causador da HIC.

A monitorização, no espaço extradural, é menos invasiva, mas a transmissão da PIC é mais difícil e exige o uso de transdutores de pressão mais sofisticados, que são aplicados diretamente sobre a duramáter. O mesmo acontece com a monitorização da PIC efetuada através da fontanela bregmática.

O sistema de monitorização mais utilizado atualmente apresenta um transdutor na extremidade de um cateter semi-rígido, que pode ser colocado no ventrículo, no parênquima e no espaço subaracnóideo e, através de uma fibra ótica, é conectado ao aparelho que registra os valores da pressão.

O valor normal da PIC é de até $15 \mathrm{mmHg}$, e, de maneira geral, as medidas terapêuticas são iniciadas quando a pressão ultrapassa $15-20 \mathrm{mmHg}$. Valores entre 20 a 40 mmHg são considerados moderadamente elevados, e acima de $40 \mathrm{mmHg}$, gravemente elevados. A PIC, muitas vezes, está elevada nos pacientes com TCE e, quando ela se mantém acima de $60 \mathrm{mmHg}$, é quase sempre fatal.

O traçado da PIC, obtido através da monitorização, pode apresentar ondas que por si só têm características patológicas. As ondas B caracterizam-se por apresentarem freqüência de 0,5 a 2 por minuto e amplitude menor que $50 \mathrm{mmHg}$. As ondas A, ou ondas em "plateau", apresentam amplitude maior que $50 \mathrm{mmHg}$, início e queda abruptos e duração mínima de cinco minutos. As ondas A e B podem ocorrer em traçados com valores basais normais ou aumentados e são decorrentes do comprometimento dos mecanismos vasomotores da microcirculação cerebral. Embora a alteração fisiopatológica que determina o aparecimento das ondas A e B seja a mesma, as ondas A traduzem um comprometimento mais grave da resistência cerebral vascular. Um traçado que não apresenta ondas de pressão e, sim, apenas variações rítmicas com a respiração, e que não responde a estímulos, como a tosse e a retenção de $\mathrm{CO}_{2}$, que normalmente aumenta a PIC, é denominado de traçado de padrão constante. Ele significa a completa falência vasomotora da microcirculação.

A monitorização da PIC é utilizada não somente para o diagnóstico da hipertensão, mas, também, pode ter valor prognóstico, especialmente nos pacientes com TCE grave, e ainda serve como parâmetro para avaliação das medidas terapêuticas empregadas para reduzir a PIC.

\section{EXAMES COMPLEMENTARES}

Radiografias Simples do Crânio - Os sinais de HIC, que são observados nas radiografias simples do crânio, são a macrocefalia e a desproporção craniofacial em lactentes, a diástase de suturas em crianças $\mathrm{e}$, às vezes, em adultos jovens, o aumento das impressões digitiformes e as erosões na sela turca. As duas últimas dependem respectivamente da pulsação acentuada dos giros corticais contra a tábua interna do crânio e da pulsação do terceiro ventrículo dilatado sobre a sela, causando desmineralização óssea. Essas alterações não aparecem nos quadros agudos de HIC.

Ocasionalmente, a radiografia simples revela calcificações patológicas (neoplasias e lesões inflamatórias) e desvio das calcificações fisiológicas (pineal e plexos coróideos). Após a introdução da Tomografia Computadorizada, sua utilidade foi muito reduzida. 
Tomografia Axial Computadorizada (TC) - A TC não permite predizer o valor da PIC, mas fornece dados indiretos muito importantes para a sua avaliação. Entre os sinais estão: presença de uma lesão expansiva, geralmente com efeito de massa (desvio de estruturas normais de sua posição original), desvio da linha média, desaparecimento dos ventrículos laterais e do terceiro ventrículo, dilatação do sistema ventricular, principalmente se acompanhado de hipodensidade ao redor (transudato periventricular), desaparecimento das cisternas perimesencefálicas e visualização de herniações intracranianas. aguda.

A TC é o exame ideal para pacientes com HIC

A TC é realizada em uma fase chamada simples e noutra posterior à injeção de contraste iodado endovenoso. A fase contrastada contribui muito no esclarecimento diagnóstio de doenças em que há quebra da barreira hematoencefálica. A partir desses dados, pode-se determinar o mecanismo fisiopatológico e o diagnóstico da causa da hipertensão.

Ressonância Nuclear Magnética (RNM) - A RNM demonstra a anatomia intracraniana de forma mais detalhada que a TC e pode fornecer melhores dados $^{(27)}$. Entretanto é um exame de alto custo e não é adequado para ser realizado em paciente em estado grave e na fase aguda (mais demorado e uso de aparelhos especiais não magnéticos para ventilação controlada).

Angiografia Cerebral - A angiografia cerebral pode ser utilizada na demonstração de doenças vasculares que podem causar HIC, como as fístulas durais arteriovenosas e obstruções das veias jugulares, além de poder ser utilizada na desobstrução de seios e veias como terapia endovascular.

Doppler Transcraniano - Variações na pressão de perfusão cerebral causam mudanças na velocidade do fluxo sangüíneo cerebral, medida pelo Doppler transcraniano, a partir dos valores das ondas de pressão sistólica e diastólica. Os valores da pressão de perfusão cerebral por este método apresentam uma diferença de 10 mmHg em relação à medida real, um valor aceitável para determinar-se a pressão de perfusão cerebral, mas elevado, quando se trata de PIC. Esse método pode ser utilizado no seguimento de pacientes em unidades de tratamento intensivo, pois medidas seriadas podem fornecer dados mais confiáveis $^{(28)}$.

\section{TRATAMENTO}

O tratamento ideal da HIC visa a remoção da sua causa. Esse objetivo pode ser alcançado em alguns pacientes que apresentam lesões expansivas, que podem ser removidas. Muitas vezes, porém, isso não é possível e, então, medidas concomitantes ou de emergência devem ser tomadas, enquanto a causa não é removida. Entre elas, estão incluídas medidas de ordem geral e medidas específicas.

\subsection{Medidas Gerais}

A posição em decúbito dorsal e com a cabeça elevada a $30^{\circ}$ melhora a drenagem venosa, a reabsorção liquórica e a ventilação. A flexão ou rotação da cabeça diminui o fluxo na jugular e aumenta a pressão intracraniana.

O paciente comatoso pode sentir dor, apesar de poder não ter resposta motora ou verbal, e a resposta vegetativa causada pela dor pode piorar o quadro de HIC. A colocação correta de sondas e coletores, identificação de doenças associadas (fraturas e doenças abdominais) são importantes para aliviar a dor.

A desobstrução de vias aéreas deve ser de curta duração e repetida, se necessário, e pode ser precedida de uma hiperventilação com o objetivo de limitar a HIC durante esse período.

Os distúrbios hidroeletrolíticos podem agravar a HIC, principalmente a hiponatremia, portanto a hidratação sempre deve ser feita, procurando a manutenção da homeostase.

Se a respiração espontânea do paciente não é suficiente para manter a $\mathrm{PO}_{2}$ acima de $60-70 \mathrm{mmHg}$ e a $\mathrm{PCO}_{2}$ arterial entre $30-40 \mathrm{mmHg}$, a ventilação mecânica deve ser instalada.

A avaliação geral do paciente e a correção de possíveis distúrbios devem ser feitos antes de medidas específicas.

\subsection{Medidas Específicas}

Inibição da produção de líquido cefalorraquidiano

Pode ser efetuada, empregando-se corticosteróides e inibidores da anidrase carbônica, como a acetazolamida.

A dexatometasona age, interferindo nos mecanismos de troca da membrana celular no plexo coróideo, inibindo a função secretória das células epiteliais.

Drogas que inibem a ação da anidrase carbônica, impedindo a hidratação do $\mathrm{CO}_{2}$, como a acetazolamida e a metazolamida, reduzem a formação de LCR a partir do $\mathrm{CO}_{2}$ produzido metabolicamente. 
Associando-se a dexametasona à acetazolamida, consegue-se uma redução de até $30 \%$ da produção liquórica, que pode ser útil no tratamento da HIC.

\subsection{Drenagem de líquido cefalorraquidiano (LCR)}

A remoção de LCR através da drenagem ventricular é um excelente procedimento para a redução da PIC aumentada, embora essa redução seja de curta duração. Em pacientes com TCE, sua utilização prática está prejudicada, porque os ventrículos, geralmente, estão diminuídos de tamanho devido ao edema cerebral, ou estão deslocados da sua posição normal por lesões expansivas intracranianas.

A drenagem liquórica pode ser definitiva nos casos de hidrocefalia, através da instalação de um sistema de derivação, geralmente, ventrículoperitoneal.

\subsection{Hiperventilação}

O efeito da hiperventilação, na diminuição da PIC, é conhecido de longa data. A redução da $\mathrm{PaCO}_{2}$ arterial pela hiperventilação determina uma alcalose, e o aumento do $\mathrm{pH}$ tem um efeito direto sobre as arteríolas, provocando vasoconstrição. A vasoconstrição determina um aumento na RVC, que impede o bombeamento de sangue para os vasos de paredes finas e a conseqüente diminuição do volume sangüíneo intracraniano e queda da PIC.

O efeito da hiperventilação sobre a PIC manifesta-se rapidamente (inicia em trinta (30) segundos e estabiliza-se em cinco minutos) e a duração é de algumas horas. Devido ao seu mecanismo de ação, esse efeito é mais intenso nos casos em que o cérebro está “apertado", na presença de inchaço cerebral. A hiperventilação está indicada, quando é necessária uma redução aguda na PIC.

Além da queda da PIC, a respiração artificial proporciona uma diminuição no consumo de oxigênio por redução da atividade muscular, uma menor tendência à atelectasia e permite o uso de sedativos que poderiam interferir com o padrão respiratório. Quando usada com pressão inspiratória final negativa, a hiperventilação melhora também o retorno venoso no segmento cefálico.

A hiperventilação deve ser utilizada para manter a $\mathrm{PaCO}_{2}$ arterial entre 25 e $35 \mathrm{mmHg}$. Valores abaixo de $20 \mathrm{mmHg}$ podem levar à hipóxia cerebral por vasoconstrição intensa, além de provocar o efeito Bohr sobre a curva de dissociação da hemoglobina, que são efeitos indesejáveis no tratamento dos pacientes.
O conceito atual da hiperventilação é o da sua adequação em relação à medida da pressão intracraniana e ao estado metabólico cerebral, avaliado pelo consumo de $\mathrm{O}_{2}$ pelo tecido cerebral, medido pela diferença de saturação de $\mathrm{O}_{2}$ do sangue arterial e do sangue venoso da veia jugular interna. Portanto, para avaliarmos os parâmetros de ventilação, devemos conhecer a pressão intracraniana e o estado de consumo de $\mathrm{O}_{2}$. Em um paciente com baixa extração de $\mathrm{O}_{2}$, interpreta-se que o fluxo sangüíneo cerebral está maior que as necessidades metabólicas, portanto pode ser diminuído pela diminuição do $\mathrm{CO}_{2}$. No caso de uma extração elevada de $\mathrm{O}_{2}$, o FSC está insuficiente, podendo haver uma aumento do fluxo pelo aumento do $\mathrm{CO}_{2}$ para evitar isquemia cerebral, se o valor da PIC monitorizada permitir ${ }^{(9,29)}$.

\subsection{Diuréticos}

As soluções diuréticas hipertônicas agem sobre a PIC através do seu efeito osmótico (partículas de alto peso molecular), o qual propicia a retirada de líquido do espaço extracelular para o intravascular. Esse efeito ocorre tanto no cérebro normal como no cérebro lesado. Os agentes osmoticamente ativos também traduzem a viscosidade sangüínea, provocando vasoconstrição reflexa e redução da PIC, e, por esse motivo, devem ser administrado em "bolus".

O manitol provoca elevação aguda na osmolaridade sangüínea, que leva a uma queda na produção e na pressão do LCR, e redução no conteúdo de água tissular. O manitol pode ser empregado em solução a $20 \%$, na dose inicial de 0,25 a $0,5 \mathrm{~g} / \mathrm{Kg}$, em infusão venosa de $7 \mathrm{ml} /$ minuto. A dose diária varia de $0,5 \mathrm{a}$ $2,0 \mathrm{~g} / \mathrm{Kg}$. A queda na PIC é observada dez (10) a vinte (20) minutos após a infusão. Quando usado com a furosemida, a queda da PIC é mais rápida e tem maior duração, indicando um efeito sinérgico entre as drogas.

As soluções hipertônicas causam hipervolemia, que pode ser um problema em pacientes idosos e cardiopatas, e diurese excessiva que pode levar à desidratação e à perda de eletrólitos, em crianças.

Devido aos efeitos rápidos sobre a PIC e aos seus efeitos colaterais, as soluções hipertônicas são empregadas para o tratamento de HIC aguda, não sendo adequadas para a utilização prolongada. O uso prolongado de manitol pode produzir hiperosmolaridade, o que o torna inefetivo, e pode estar associado com insuficiência renal aguda. Em pacientes em uso repetido de manitol, a osmolaridade não deve ultrapassar 
$320 \operatorname{mosm} / \mathrm{Kg}$. Além da osmolaridade, níveis séricos altos contínuos de manitol podem aumentar a passagem da droga do espaço intra para o extravascular do cérebro lesado e causar o efeito rebote, ou seja, um aumento da PIC acima dos níveis anteriores, após a queda inicial. Entretanto esse efeito, geralmente, não é acompanhado por alterações clínicas.

Outros diuréticos não osmóticos podem agir na PIC. A furosemida tem sido empregada freqüentemente no tratamento de pacientes com HIC. Ela age na redução da PIC, em parte, por inibir em $33 \%$ a produção liquórica, o que é independente do seu efeito diurético. Existem evidências de que a furosemida só age quando utilizada juntamente com diuréticos osmóticos $^{(30 / 36)}$.

\subsection{Glicocorticóides}

Provocam a redução da PIC através de mecanismos não totalmente esclarecidos. Um dos seus efeitos é a estabilização das membranas celulares, restabelecendo o mecanismo de transporte ativo e permitindo a correção dos distúrbios que propiciam a formação e/ou aumento do edema cerebral, especialmente o vasogênico. Outros efeitos observados são a redução na produção de LCR e possível melhora da função neurológica, independentemente da melhora do edema cerebral.

A dexametasona, empregada em doses de 16 a $24 \mathrm{mg} / \mathrm{dia}$, em pacientes portadores de metásteses intracranianas, determina uma diminuição da PIC após dois a oito dias de uso, embora a melhora clínica ocorra mais rapidamente.

O uso de glicocorticóides pode ocasionar o aparecimento de vários efeitos colaterais, dos quais os mais importantes são o retardo na cicatrização das feridas, a diminuição da resistência a infecções, sangramento gastrintestinal, síndrome de Cushing e depressão da supra-renal após o uso prolongado.

O efeito dos glicocorticóides no tratamento dos pacientes com TCE, em que ocorrem lesões anatômicas diretas da barreira hematoencefálica, que determinam a formação de edema cerebral por vários mecanismos, é motivo de controvérsia na literatura. Entretanto, há um consenso segundo o qual os glicocorticóides não são efetivos no tratamento da HIC e não melhoram o prognóstico de pacientes com TCE, e, por isto, progressivamente a sua utilização tem sido reduzida nesses $\operatorname{casos}^{(30)}$.

\subsection{Barbitúricos}

Os barbitúricos de ação rápida (tionembutal, pentobarbital, tiopental) agem agudamente (um a dois minutos) na redução da PIC, provocando diretamente vasoconstrição das arteríolas cerebrais. Cronicamente, agem através do aumento do tônus muscular arterial, da redução da pressão hidrostática nas áreas lesadas, da diminuição da pressão arterial média (PAM), da redução das variações da PIC a estímulos nocivos e da redução do metabolismo cerebral com diminuição do consumo de oxigênio.

O efeito dos barbitúricos sobre a PIC é obtido com doses que determinam o coma iatrogênico e, em tal situação, a indicação do tratamento, bem como a sua manutenção, exigem uma série de cuidados: deve ser indicado apenas para pacientes com alterações importantes no nível de consciência, quando o aumento da PIC não respondeu às medidas terapêuticas anteriores, e quando foi afastada a presença de um processo expansivo intracraniano, monitorização da PAM, monitorização da PIC e respiração controlada por ventiladores mecânicos.

A dosagem de pentobarbital empregada é de 3 a $5 \mathrm{mg} / \mathrm{Kg}$ administrada EV em "bolus", como dose de ataque. Essa dose pode ser repetida após quinze (15) minutos, se não houver resposta (queda da PIC). A dose de manutenção é de 100 a 200 mg/hora. A concentração sérica deve ser mantida entre 2,5 a $3,5 \mathrm{mg} \%$ e controlada através de dosagens diárias. $\mathrm{O}$ seu uso deve ser por pelo menos setenta e duas (72) horas.

A suspensão do barbitúrico deve ser efetuada lentamente (quatro (4) a cinco (5) dias), quando a normalização da PIC pode ser observada.

Entre os efeitos colaterais dos barbitúricos, destaca-se o próprio coma iatrogênico, que dificulta a avaliação da progressão da lesão neurológica através do exame clínico. Esse problema deve ser contornado com a realização prévia de TC e com a repetição do exame sempre que alterações pupilares ou dos valores da PIC o justificarem. Outros efeitos colaterais, observados e que determinam a suspensão do uso são a PAM abaixo de $70 \mathrm{mmHg}$, em adultos, e $50 \mathrm{mmHg}$, em crianças, alterações no débito cardíaco e hipóxia inexplicada.

Embora os barbitúricos propiciem uma melhora aparente na evolução inicial dos pacientes com TCE, diminuindo a necessidade do uso do manitol para reduzir a PIC, estudos prospectivos mostram que não há evidência de melhora do prognóstico desses pacientes na evolução a longo prazo ${ }^{(30)}$. 


\subsection{Solução Salina Hipertônica}

O efeito da solução salina hipertônia (SSH) na redução do volume cerebral, é conhecido, há vários anos, mas a avaliação na redução da PIC passou a ser estudada a partir dos anos oitenta (80). A SSH é um efetivo agente osmótico, que cria uma força para atrair a água do interstício e espaço intracelular do cérebro para o compartimento intravascular. A redução do volume de água cerebral causa diminuição da pressão intracraniana. $\mathrm{O}$ cloreto de sódio tem um maior coeficiente de reflexão que o manitol. O coeficiente de reflexão é definido como a habilidade da barreira hematoencefálica para excluir uma substância. A osmolaridade da solução salina 23,4\% (8008 mOsm/L) é seis vezes maior que a do manitol $(1372 \mathrm{mOsm} / \mathrm{L})$. Esses dados podem significar que a SSH é melhor que o manitol ${ }^{(37)}$.

A SSH pode ser usada em "bolus" de $30 \mathrm{ml}$, durante quinze (15) a vinte (20) minutos, por um ca- teter venoso central, quando a HIC é detectada. Essa dose é equimolecular, a 0,5 a $1,0 \mathrm{~g} / \mathrm{Kg}$.

\subsection{Hipotermia}

A hipotermia tem o efeito de diminuir a pressão intracraniana, do fluxo sangüíneo cerebral e do consumo de $\mathrm{O}_{2}$ pelo cérebro. A aplicação dessa metodologia foi abandonada devido às complicações clínicas, principalmente infecciosas, oriundas da baixa temperatura.

No início dos anos noventa (90), surgiram trabalhos, mostrando que a hipotermia moderada $\left(34^{\circ} \mathrm{C}\right)$ leva a benefícios importantes em relação ao tratamento da HIC, sem aumentar significativamente as complicações clínicas.

A hipotermia é efetiva na prevenção do aumento da PIC, no intervalo de 20 a $40 \mathrm{mmHg}$, quando as medidas convencionais já foram tomadas. Pacientes com ingurgitamento difuso não respondem à hipotermia moderada, sendo que pacientes com lesões focais evoluem bem ${ }^{(38)}$.

CARLOTTI JR CG; COLLI BO \& DIAS LAA. Intracranial hypertension. Medicina, Ribeirão Preto, 31: 552-562, oct./dec. 1998.

ABSTRACT: The relation between the volume of the brain and the skull determines the intracranial pressure, that has as reference the atmospheric pressure. In normal conditions the intracranial pressure has oscillations determined by the breathing and heart cycles. Several pathologies determine the increase of the intracranial pressure, the most frequent the head trauma.

Should be valued for the diagnosis of the intracranial hypertension $(\mathrm{ICH})$ the clinical picture of headache, vomiting and papilledema. The most important subsidiary exams are the image methods mainly the Computed Tomography (CT) and the Magnetic Resonance Imaging (MRI).

Monitorization of the intracranial pressure is important for severe cases of ICH. Several therapeutic modalities can ben used, as hypocarbia induced by hyperventilation, osmotics diuretics, barbiturates, hypothermia and special general measures with the patient.

UNITERMS: Intracranial Pressure. Diagnosis.

\section{REFERÊNCIAS BIBLIOGRÁFICAS}

1 - MARMAROU A \& TABADDOR K. Intracranial pressure: physiology and pathophysiology. In: COOPER PR, ed. Head injury. $3^{\text {th }}$ ed. Willians \& Wilkins, Baltimore, p. 159-176, 1993

2 - LEE KR \& HOFF JT. Intracranial pressure. In: YOUMANS JR, ed: Neurology surgery. $4^{\text {th }}$ ed. W.B. Saunders, Philadelphia, v. 1, p 491-518, 1996.

3 - MILHORAT TH et al. Cerebrospinal fluid production by the choroid plexus and brain. Science 173: 330-332, 1971.

4 - VOGH BP \& MAREN TH. Sodium, chloride and bicarbonate movement from plasma to cerebrospinal fluid in cats. Am J Physiol 228: 673-683, 1975.
5 - BAKAY ERA \& WOOD JH. Pathophysiology of cerebrospinal fluid in trauma. In: BECKER D \& POVLISHOCK J: Central nervous system trauma status report. National Institute of Health, New York, p. 89-137, 1985.

6 - POLLAY M. Review of spinal fluid physiology: Production and absortion in relation to pressure. Clin Neurosurg 24: 254-269, 1997.

7 - HALSEY JR JH et al. Regional cerebral blood flow comparison of right and left hand movement, Neurology 29: 21-28, 1979.

8 - MUIZELAAR JP \& OBRIST WD. Cerebral blood flow and brain metabolism with brain injury. In: BECKER D \& POVLISHOCK $\mathrm{J}$ : Central nervous system trauma status report. National Institute of Health, New York, p. 123-137, 1985. 
9 - CRUZ J. Hemometabolismo cerebral: de medidas isoladas a medidas de monitorização e terapêutica. Arq Neuropsiquiatr 51: 1-7, 1993.

10 - LE ROUX PD et al. Cerebral arteriovenous oxygen difference: a predictor of cerebral infarction and outcome in patients with severe head injury. J Neurosurg 87: 1-8, 1997.

11 - COGNARD C et al. Dural arteriovenous fistulas as a cause of intracranioal hypertension due to impairment of cranial venous outflow. J Neurol Neurosurg Psychiatry 65: 308-316, 1998.

12 - DUKE BJ et al. Traumatic bilateral jugular vein thrombosis: case report and review of the literature. Neurosurgery 41: 680-683, 1997.

13 - KARAHALIOS DG et al. Elevated intracranial venous pressure as a universal mechanism in pseudotumor cerebriare of varying etiologies. Neurology 46: 198-202, 1996.

14 - SOLER D et al. Diagnosis and management of benign intracranial hypertension. Arch Dis Child 78: 89-94, 1998.

15 - SUGERMAN HJ et al. Increased intra-abdominal pressure and cardiac filling pressure in obesity-associated pseudotumor cerebri. Neurology 49: 507-511, 1997.

16 - POPP J \& BOURKE RS. Cerebral edema: etiology, pathophysiology and therapeutic considerations. Contemp Neurosurg 1: 1-6, 1977.

17- ASATO R et al. Chronological sequences and blood-brain barrier permebeality changes in local injury as assessed by nuclear magnetic resonance (NMR) images from sliced rat brain. Stroke 14: 191-196, 1983.

18 - COLLI BO. Hipertensão intracraniana: fisiopatologia, diagnóstico e tratamento. J Bras Neurocirurg 2: 25-34, 1990.

19 - SHIGENO T et al. The determination of brain water content: microgravimetry versus drying-weighing method. $\mathbf{J}$ Neurosurg 57: 99-108, 1982.

20 - ASTRUP J. Energy-requiring cell functions in the ischemic brain. Their critical supply and possible inhibition in protective therapy. J Neurosurg 56: 482-498, 1982.

21 - LANGFITT TW; WEINSTEIN JD \& KASSELL NF. Vascular factors in head injury: contribuition to brain swelling and intracranial hypertension. In: CAVANESS WF \& WALKER AE. Head injury: Conference Proceedings. JB Lippincott, Philadelphia, p. 172-194, 1966.

22 - BARRAQUER-BORDAS L. Síndrome de hipertensión endocraneana. In: BARRAQUER-BORDAS L: Neurologia fundamental. $2^{\mathrm{a}}$ ed. Ediciones Toray, Barcelona, p. 309-323, 1968.

23 - ORIOT D \& NASSIMI A. Hypertension intracrânienne de l'enfant: de la physiopathologie à la prise en charge thérapeutique. Arch Pédiatr 5: 773-782, 1998.
24 - PLUM F \& POSNER JB. The pathological physiology of signs and symptons of coma. In: PLUM F \& POSNER JB: The diagnosis of stupor and coma. $2^{\text {nd }}$ ed., F.A. Davis, Philadelphia, p 1-61,1978.

25 - BRUCE DA et al. Regional cerebral blood flow, intracranial pressure, and brain metabolism in comatose patients. $\mathbf{J}$ Neurosurg 38: 131-144, 1973.

26 - KELLY DF et al. Hyperemia following traumatic brain injury: relationship to intracranial hypertension and outcome. J Neurosurg 85: 762-771, 1996.

27 - BELL BA et al. Brain water measured by magnetic resonance imaging. Lancet 1: 66-69, 1987.

28 - CZOSNYKA M et al. Cerebral perfusion pressure in headinjured patients: a noninvasive assessment using transcranial Doppler ultrasonography. J Neurosurg 88: 802-808, 1998.

29 - OBRIST WD et al. Relation of cerebral blood flow to neurological status and outcome in head injured patients. $J$ Neurosurg 51: 292-300, 1979.

30 - CHESNUT RM; MARSHALL LF \& MARSHALL SB. Medical management of intracranial pressure. In: COOPER PR. Head injury $3^{\text {th }}$ ed, Willians \& Wilkins, Baltimore, p. 225-246, 1993.

31 - MARSHALL LF et al. Mannitol dose requirements in braininjured patients. J Neurosurg 48: 169-172, 1978.

32 - MUIZELAAR JPO et al. Mannitol causes compensatory cerebral vasoconstriction and vasodilatation in response to blood viscosity changes. J Neurosurg 59: 822-828, 1983.

33 - NATH F \& GALBRAITH S. The effect of mannitol on cerebral white matter water content. J Neurosug 65: 41-43, 1986.

34 - SANSON D \& BEYER CW JR. Furosemide in the intraoperative reduction of intracranial pressure in the patient with subarachnoid hemorrhage . Neurosurgery 10: 167-169, 1982.

35 - WILKINSON HA \& ROSENFELD S. Furosemide and mannitol in treatment of acute experimental intracranial hypertension. Neurosurgery 12: 405-410, 1983.

36 - WILKINSON HA; WEPSIC JG \& AUSTIN G. Diuretic synergy in the treatment of acute experimental cerebral edema. J Neurosurg 34: 203-208, 1971.

37 - SUAREZ JI et al. Treatment of refractory intracranial hypertension with 23,4\% saline. Crit Care Med 26: 1118-1122, 1998.

38 - SHIOZAKI T et al. Selection of severely head injured patients for mild hypothermia therapy. J Neurosurg 89: 206-211, 1998.

Recebido para publicação em 04/03/98

Aprovado para publicação em 07/10/98 